\title{
BGP expression in gastric biopsies may predict the development of new lesions after local treatment for early gastric cancer
}

\author{
Shinya Shimada ${ }^{1,2}$, Kenji Shiomori $^{1}$, Ubehiko Honmyo ${ }^{1}$, Masanobu Maeno ${ }^{1}$, Yasushi Yagi ${ }^{3}$, \\ and Michio OGAWA ${ }^{1}$ \\ ${ }^{1}$ Second Department of Surgery, Kumamoto University School of Medicine, 1-1-1 Honjo, Kumamoto 860-8556, Japan \\ ${ }^{2}$ Department of Surgery, Yatsushiro Health Insurance General Hospital, Yatsushiro, Japan \\ ${ }^{3}$ Department of Surgery, Kumamoto Regional Hospital, Kumamoto, Japan
}

\begin{abstract}
Background. Our previous studies have demonstrated the significant role of the generative cells of intestinal metaplasia (IM) expressing brain (fetal)-type glycogen phosphorylase (BGP) (BGP-IM) as a premalignant lesion of intestinal-type adenocarcinoma. The aims of the present study were to investigate the incidence of BGP-IM in gastric biopsy specimens and to establish BGP-IM as a predictor of the coexistence of accessory carcinoma and/or metachronous cancers before and after local treatment for early gastric carcinoma.

Methods. We studied the incidence of BGP-IM in eight endoscopic biopsy specimens of methylene blue-positive mucosa of the stomach obtained from patients with multiple gastric carcinomas $(n=14)$, a single carcinoma $(n=25)$, and atrophic gastritis $(n=20)$.

Results. BGP positivity was $93.3 \%$ in the multiple carcinomas and $80.0 \%$ in the single carcinomas. The incidences of BGPIM (mean percentage \pm SD) in the stomachs with multiple carcinomas, single carcinoma, and atrophic gastritis were $83.2 \% \pm 22.8 \%, 36.5 \% \pm 41.3 \%$, and $7.1 \% \pm 18.0 \%$, respectively. The incidence was significantly higher in the stomachs with multiple carcinomas than in those with a single carcinoma or those with atrophic gastritis $(P<0.001)$.

Conclusion. It is suggested that the frequent appearance of BGP-IM reflects the high potential of carcinogenesis of intestinal-type gastric cancer, and that the involvement of BGP-IM in more than $50 \%$ of the eight biopsies may be a predictor of the coexistence of accessory and/or metachronous carcinoma before and after local treatment for early gastric carcinoma.
\end{abstract}

Key words Gastric carcinoma - Endoscopic mucosal resection · Local treatment $\cdot$ Intestinal metaplasia $\cdot$ Glycogen phosphorylase $\cdot$ Predictor

Offprint requests to: $\mathrm{M}$. Ogawa

Received: January 10, 2002 / Accepted: March 26, 2002

\section{Introduction}

The recent advent of endoscopic and laparoscopic local treatments has offered a better quality of life to patients with early gastric carcinoma involving no lymph node metastasis [1-5]. These treatments, however, incur increasing risks of missing the coexistence of accessory (microscopic) carcinomas and/or developing new cancers in the remnant stomach [6-9].

The incidence of multiple primary gastric carcinoma has been reported to be from $5 \%$ to $10 \%$ in patients who had gastrectomy for gastric cancer [10-14]. The incidence is elevated with age and male sex, and with intestinal-type tumors; frequent occurrence in the lower third, and mucosal cancers, were significantly correlated with multiple early gastric cancer. However, these accessory lesions were missed preoperatively in approximately $30 \%-40 \%$ of the patients with multifocal early gastric cancers. Furthermore, considerable numbers of microscopic cancers could have been overlooked. Almost all of these unrecognized lesions, however, should be co-resected by distal gastrectomy, because those concomitant lesions were detected significantly more frequently in the lower part of the stomach on the distal side of the atrophic border than in the upper part $[15,16]$. Also, microscopic cancers may grow into clinically significant lesions within several years when a gastric carcinoma is removed by local treatment [9]. In addition, it has been reported that the histological types of these carcinomas are almost all of the intestinal type. Therefore, we should always remember that other gastric lesions may also be present and/or grow when we are treating patients with gastric cancer by local treatment such as endoscopic mucosal resection (EMR) or laparoscopic wedge resection.

Local treatment for early gastric cancer is currently indicated mainly for intestinal-type carcinoma. If there were some indicators that predicted the frequent coexistence of multiple gastric cancers (including micro- 
scopic carcinoma) and/or the metachronous growth of another gastric cancer of the intestinal type, these would be very useful to identify the high-risk group and would contribute to the follow-up studies after local treatment of gastric cancer.

We have previously demonstrated the frequent abnormal expression of brain (fetal)-type glycogen phosphorylase (BGP) in gastric carcinoma, especially in intestinal-type cancer [17-21]. Furthermore, with regard to the carcinogenesis of gastric carcinoma, we have also pointed out the significant role of the generative cells of intestinal metaplasia (IM) expressing BGP (BGP-IM) as a premalignant lesion of intestinal-type adenocarcinoma.

In the current study, using gastric biopsy specimens, BGP expression in IM was evaluated as a predictive indicator showing the coexistence of accessory carcinoma of the human stomach, and as a possible predictor of metachronous gastric carcinoma.

\section{Patients and methods}

\section{Patients and specimens}

Between 1997 and 2000, 59 patients with intestinal-type early gastric cancer and endoscopic atrophic gastritis were analyzed in this study. Of these patients, 14 had synchronous multiple gastric carcinomas, 25 had a single cancer, and 20 had endoscopic atrophic gastritis without any localized lesions. During endoscopic examination, the lower two-thirds of the stomach was dyed with methylene blue $[22,23]$ and eight endoscopic biopsies were made from the stained mucosa (Fig. 1) in the anterior, posterior, greater and lesser curvature wall of the antrum and lower body of the stomach, respectively. Informed consent was obtained from all the patients.

The biopsy specimens were put on small filters, fixed in $10 \%$ buffered formaldehyde for 1 day, embedded in paraffin to make longitudinal sections of gastric mucosa including the generative cells of IM, and cut into three serial sections for histological and immunohistochemical examinations.

\section{Antibody}

Antibody against human BGP was raised as previously reported by Ignacio et al. [24], with modification. Briefly, a synthesized 13-residue peptide (CDLQIPPPNIPRD) corresponding to cystein coupled to the 12-carboxyterminal residues of BGP, was chosen for the immunogen, and this had no significant homology with other protein including human liver and muscle-type GP, determined using Gene Work's homol-

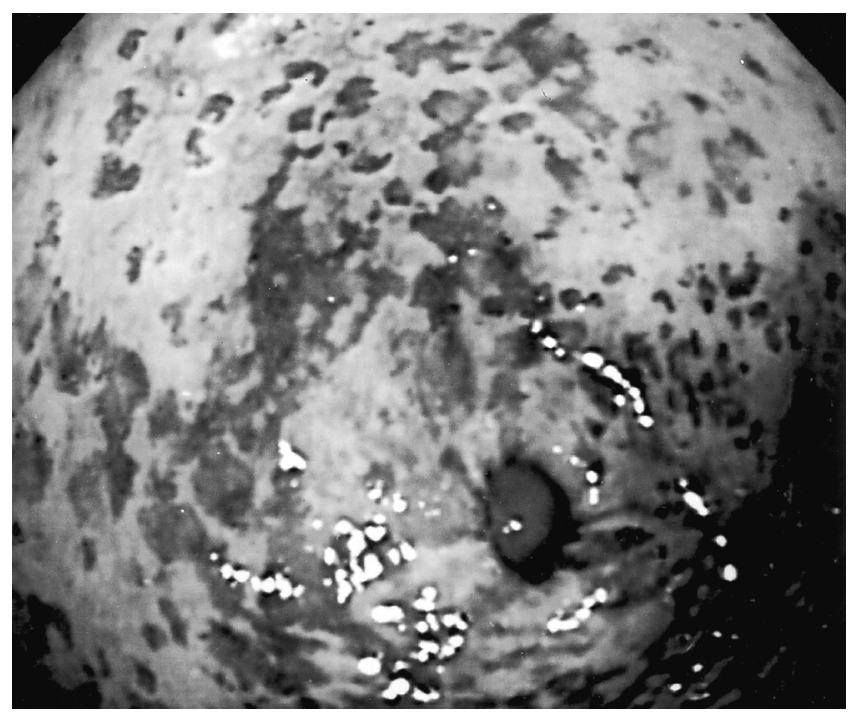

Fig. 1. Endoscopic dye staining of the stomach using methylene blue. Intestinal metaplasia was macroscopically visualized as blue islands in the gastric mucosa

ogy search. The N-terminal cysteine of the peptide was coupled with activated keyhole limpet hemocyanin (KLH; Pierce, Rockford, IL, USA). Adult rabbits were immunized with four subcutaneous injections at 1-week intervals, with $100 \mu \mathrm{g}$ of the KLH-peptide with Freund's complete or incomplete adjuvant. One week after the last injection, the same amount of the KLH-peptide was injected subcutaneously as a booster, and 1 week later, blood samples were collected from the jugular vein. Immunoglobulin $\mathrm{G}(\mathrm{IgG})$ fractions of pooled antisera (approximately $100 \mathrm{ml}$ ) were precipitated by adding saturated $\left(\mathrm{NH}_{4}\right)_{2} \mathrm{SO}_{4}(50 \%$ saturation $)$, dissolved in $100 \mathrm{ml}$ of phosphate-buffered saline (PBS) and dialyzed extensively in the same buffer. Part of this $\mathrm{IgG}$ fraction $(2 \mathrm{ml})$ was then applied to a column of BGP-coupled Sepharose (1 mg of the BGP peptide was coupled with $1 \mathrm{ml}$ of Hi Trap NHS-activated Sepharose) (Pharmacia Biotech, Uppsala, Sweden) at a flow rate of $0.5 \mathrm{ml} / \mathrm{min}$ at room temperature. The column was washed successively with buffer $\mathrm{A}(0.5 \mathrm{M}$ ethanolamine, $0.5 \mathrm{M} \mathrm{NaCl}, \mathrm{pH} 8.3)$ and buffer $\mathrm{B}(0.1 \mathrm{M}$ acetate buffer, $0.5 \mathrm{M} \mathrm{NaCl}, \mathrm{pH} 4.0)$. Then the antibodies bound in the column were eluted with $0.1 \mathrm{M}$ glycine- $\mathrm{HCl}$ buffer, $\mathrm{pH} 2.5$, containing $1 \mathrm{M}$ $\mathrm{NaCl}$. Fractions containing antibodies, which were detected by measuring the absorbency at $280 \mathrm{~nm}$, were collected, neutralized immediately with $1 \mathrm{M} \mathrm{NaOH}$, and dialyzed against PBS buffer.

\section{Immunohistochemistry}

ABC Elite kits (Vector Laboratories, Burlingame, CA, USA) for rabbit IgG were used. Sections of formalin-

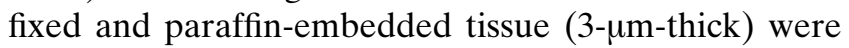


deparaffinized and hydrated through xylene and graded ethanol. The sections were incubated with normal horse serum for $30 \mathrm{~min}$ following the blocking of endogenous peroxidase activity with $0.3 \% \mathrm{H}_{2} \mathrm{O}_{2}$ in methanol for $30 \mathrm{~min}$, incubated overnight at $4{ }^{\circ} \mathrm{C}$ with optimally diluted primary antibody, and subsequently incubated with biotinylated anti-rabbit and anti-mouse antibody and avidin-biotin peroxidase complex for $30 \mathrm{~min}$ at room temperature. They were washed in $10 \mathrm{mM}$ PBS ( $\mathrm{pH}$ 7.2) between each incubation step. The sites of peroxidase binding were visualized by the diaminobenzidine method. The sections were counterstained with hematoxylin for microscopic examination. The working dilutions of the primary antibodies employed in this study were $1 \mu \mathrm{g} / \mathrm{ml}$ of the affinity purified anti-BGP. As a negative control, nonimmunized rabbit IgG was used instead of the primary antibody.

\section{Statistics}

The incidence of BGP-IM per patient was expressed as the percentage of BGP-IM appearance in the eight biopsy specimens, excluding the specimen without the generative cells of IM. Statistical analyses were performed using Student's $t$-test and Fisher's exact probability test.

\section{Results}

\section{Clinicopathological features of synchronous gastric carcinoma}

Table 1 shows the clinicopathological data of the patients. The patients with multiple early gastric carcinomas were significantly older than those with single early gastric carcinoma $(P=0.041)$. However, there was no significant difference between the patients with single carcinoma and those with atrophic gastritis. The incidence of multiple gastric carcinomas had a tendency to be higher in males than that of single carcinoma or that of atrophic gastritis.

\section{BGP expression in endoscopic biopsy specimens of gastric carcinoma and IM}

The anti-BGP antibody against specific peptide of BGP demonstrated good reactivity in the conventional paraffin sections at a low antibody concentration. Furthermore, no immunohistochemical staining of BGP was observed in normal gastric mucosa, even in the proliferating zone. The reactivity of gastric carcinoma to antiBGP antibody in endoscopic biopsy specimens is shown in Fig. 2. Strongly positive reactivity was observed in the cytoplasm of cancer cells. In $93.3 \%$ (28/30) of the multiple carcinomas and $80.0 \%(20 / 25)$ of the single carcinomas, the biopsy specimens showed positive staining for BGP. The percentage of immunohistochemical positivity for anti-BGP antibody in the intestinal-type carcinoma corresponded well with previous reports $[18,19]$. Figure 3 shows BGP-IM in a biopsy specimen. The IM glands had structural deformity to a slight degree, but no cellular atypia. The generative cell zone of IM showed positive reactivity. Strong reactivity, similar to that in the cancer cells, was observed in the cytoplasm of the generative cells of IM.

\section{Incidence of BGP-IM in stomachs with multiple carcinoma, single carcinoma, and atrophic gastritis}

As shown in Fig. 4, the distribution of the plots showing BGP-IM positivity in the stomach was extremely char-

Table 1. Clinicopathological factors in patients with multiple and single early gastric carcinoma and atrophic gastritis

\begin{tabular}{lcccc}
\hline & $\begin{array}{c}\text { Multiple; } \\
n=14 \\
(30 \text { lesions })\end{array}$ & $\begin{array}{c}\text { Single; } \\
n=25\end{array}$ & $\begin{array}{c}\text { Atrophic } \\
\text { gastritis; } \\
n=20\end{array}$ & $P$ value \\
Factors & $72.5 \pm 9.3^{*}$ & $64.0 \pm 13.3^{*}$ & $63.0 \pm 18.4$ & $0.041^{*}$ \\
\hline $\begin{array}{l}\text { Age (years }) \\
\text { Sex }\end{array}$ & $10(71.4)$ & $15(60.0)$ & $10(50.0)$ & NS \\
$\quad$ Male & $4(28.6)$ & $10(40.0)$ & $10(50.0)$ & NS \\
Female & $6(20.0)$ & $4(16.0)$ & NA & \\
Macroscopic type & $24(80.0)$ & $21(84.0)$ & NA & NS \\
$\quad$ Elevated & $16(53.3)$ & $16(64.0)$ & NA & \\
Depressed & $14(46.7)$ & $9(36.0)$ & NA & NS \\
$\quad \begin{array}{l}\text { Mumor location } \\
\text { Lower }\end{array}$ & $28(93.3)$ & $20(80.0)$ & & \\
BGP positivity & & & & \\
\hline
\end{tabular}

Values in parentheses are percentages

NA, Not applicable; NS, not significant; BGP, brain (fetal)-type glycogen phosphorylase 

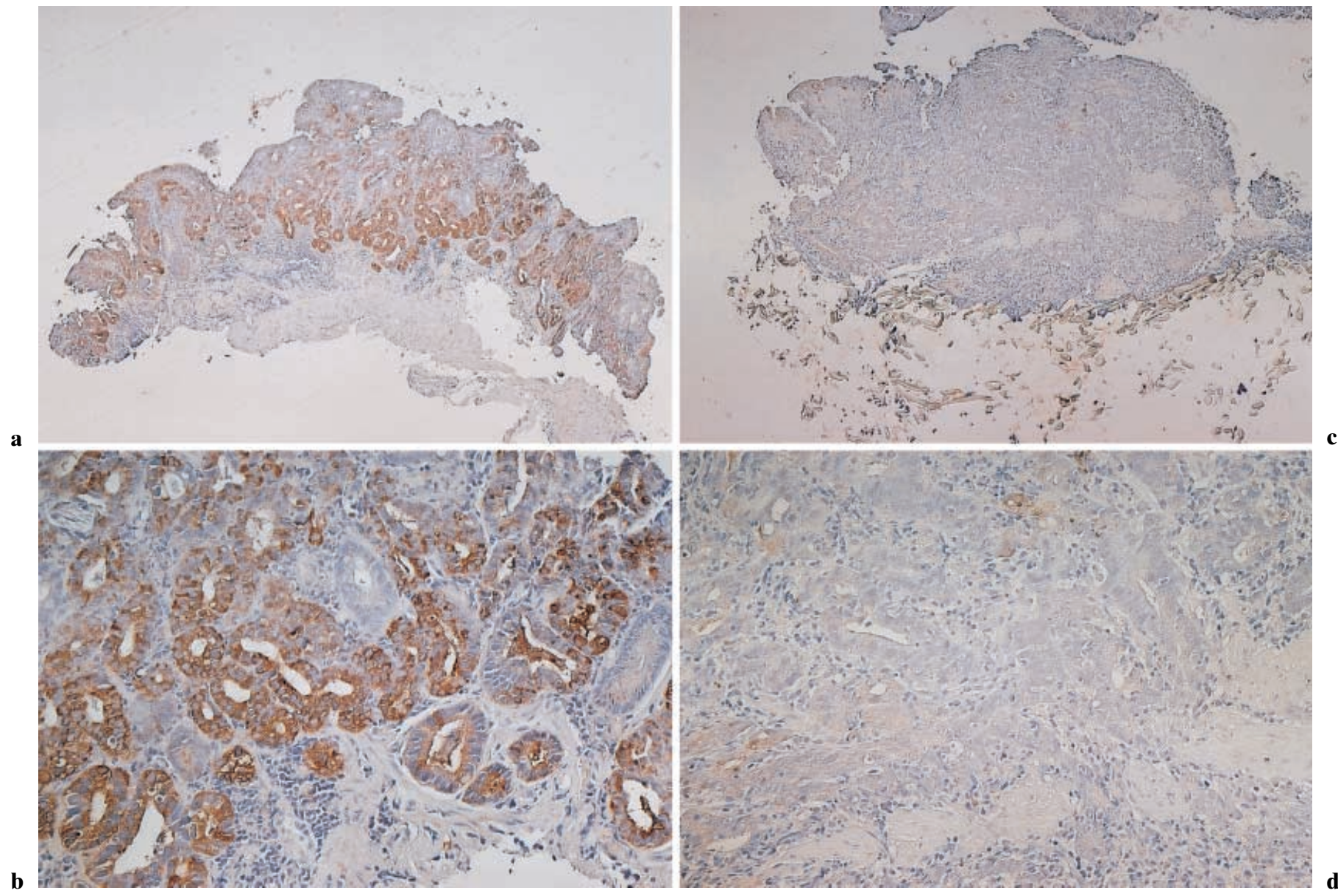

Fig. 2a-d. Immunohistochemical staining of biopsy specimens from gastric cancer foci with affinity-purified anti-brain type glycogen phosphorylase (BGP) antibody. a BGP-positive carcinoma and $\mathbf{b}$ high-power view; $\mathbf{c}$ BGP-negative carcinoma, and $\mathbf{d}$ high-power view. Strongly positive reactivity was observed in the cytoplasm of cancer cells, but none of the staining was detected in the nonneoplastic gastric gland $(\mathbf{b})$. $\mathbf{a} \times 60 ; \mathbf{b} \times 260 ; \mathbf{c} \times 60 ; \mathbf{d} \times 260$

acteristic in each group. The distribution was almost symmetrical in the multiple carcinoma and the atrophic gastritis groups. Although almost all stomachs with atrophic gastritis had no BGP-IM in any biopsy specimen, all the stomachs with multiple carcinoma had BGP-IM in each of the biopsy specimens. Furthermore, all the carcinomas in the multiple carcinoma group had high percentages of BGP-IM appearance, except for two in which BGP was negative in the cancer foci. On the other hand, a bipolarized distribution of the plots was observed in the single-carcinoma group; that is, about a quarter of the group had BGP-IM at high percentages, but about half of the group did not have it at all. The incidences of BGP-IM (mean percentage \pm $\mathrm{SD}$ ) in the stomachs with multiple carcinomas, single carcinoma, and atrophic gastritis were $83.2 \% \pm 22.8 \%$, $36.5 \% \pm 41.3 \%$, and $7.1 \% \pm 18.0 \%$, respectively (Fig. 4). The incidence of BGP-IM in the stomachs with multiple carcinomas was significantly higher than that in those with a single carcinoma $(P<0.001)$ or those with atrophic gastritis $(P<0.001)$. The incidence in stomachs with a single carcinoma was significantly higher than that in those with atrophic gastritis $(P=0.011)$.

\section{Discussion}

One of the major problems with the local treatment of gastric cancer is that of the metachronous carcinomas in other parts of the stomach being different from the initial site of the carcinoma. A recent molecular biological study has suggested that high microsatellite instability in gastric tumors had a relationship with synchronous and/or metachronous gastric cancer compared with single carcinoma, whereas there was no difference in proliferative ability, carcinogenetic pathway through p53 or K-ras, and various mismatch repair genes, although the mechanism was unclear [25]. How- 


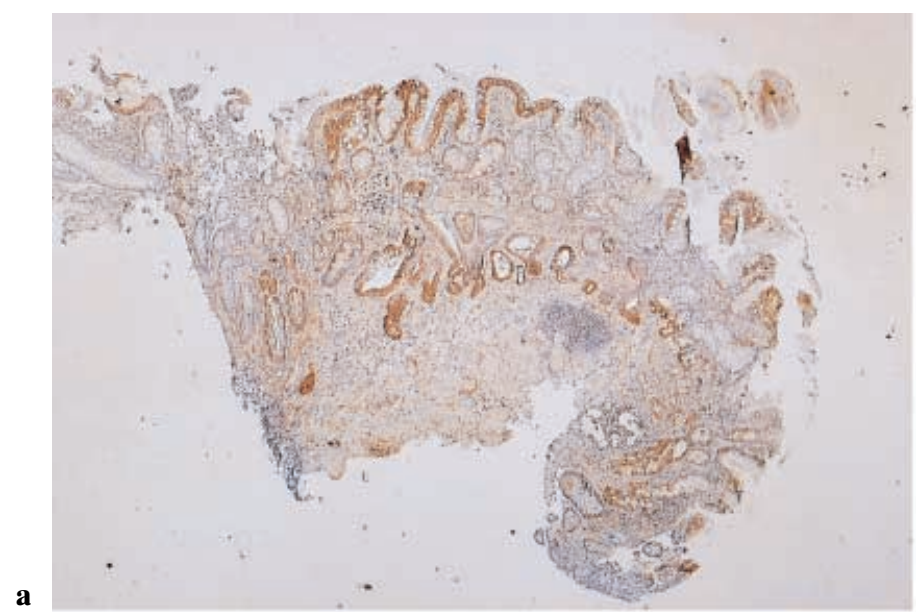

b

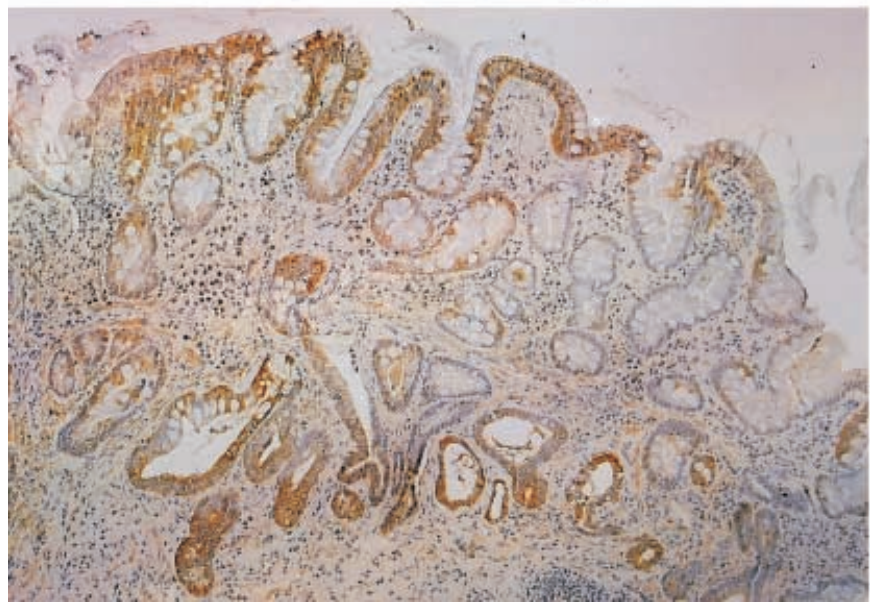

Fig. 3a-d. Immunohistochemical staining of biopsy specimens from gastric mucosa stained by methylene blue dye with affinity-purified anti-brain type glycogen phosphorylase (BGP) antibody. a BGP-positive intestinal metaplasia and $\mathbf{b}$ high-power view; c BGP-negative intestinal metaplasia and $\mathbf{d}$

ever, the application of molecular genetics in the screening and surveillance of patients for gastric carcinoma is still in its infancy. Arima et al. [8] reported that metachronous recurrence was found in 6 of 76 endoscopically treated patients, and it was detected significantly more frequently in patients whose synchronous multiple lesions were found during the initial treatment; they stressed the importance of the detection of gastric mucosal recurrence by frequent periodic endoscopic examinations during the follow-up period after the endoscopic treatment. Early detection of the metachronous cancer is beneficial for the subsequent treatment of the new lesion, for which minimally invasive therapy, including EMR, can be used. The necessity for frequent endoscopic follow-up, however, affects the quality of life for the patient and increases the overall medical cost. Therefore, a reliable predictive indicator of patients with a high risk of metachronous recurrence
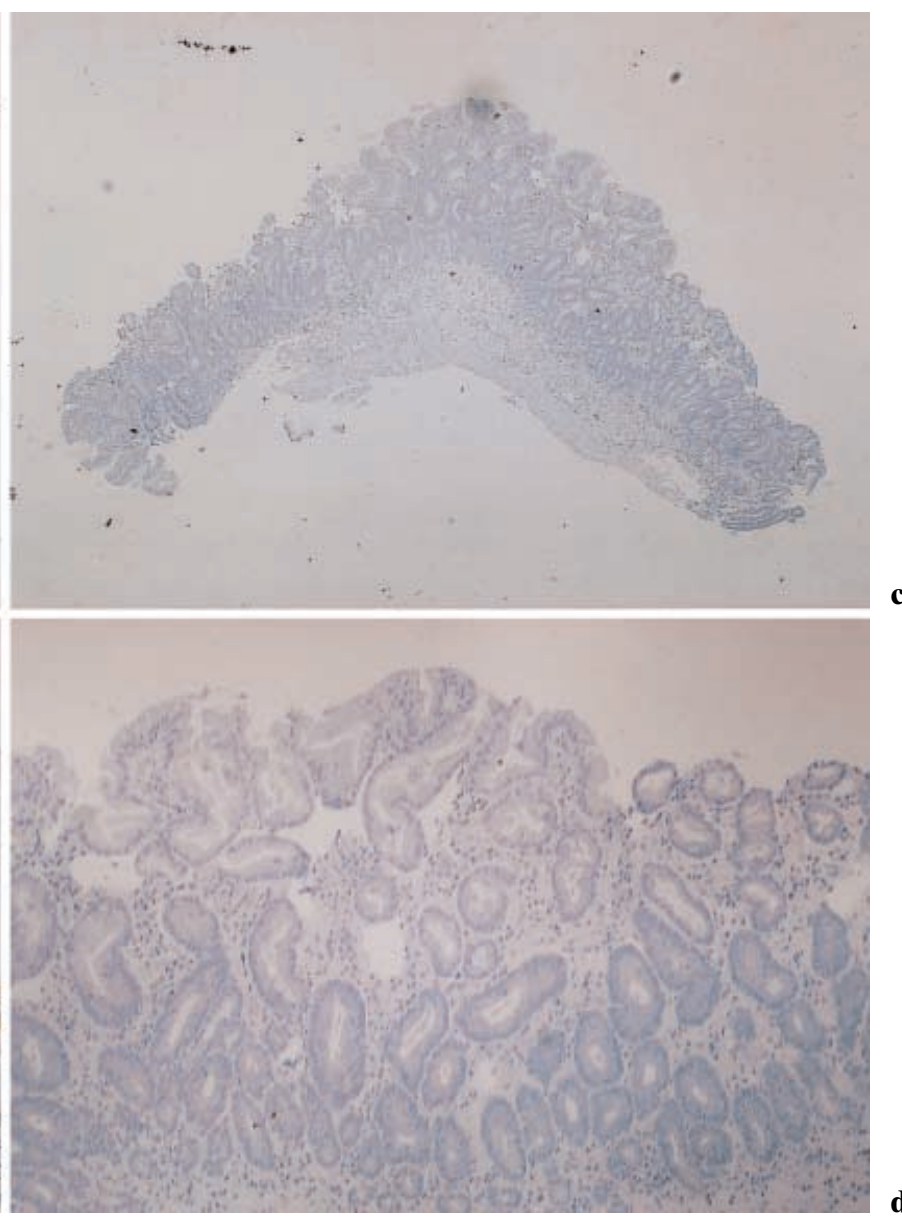

high-power view. Strongly positive reactivity was observed in the generative cells of intestinal metaplasia and its epithelium, but none of the staining was detected in the neighboring normal gastric mucosa (pyloric gland). $\mathbf{a} \times 60$; $\mathbf{b} \times 230$; $\mathbf{c} \times 60$; d $\times 230$

is very important for determining the schedule of endoscopic follow-up after the initial endoscopic treatment. Because metachronous recurrence was detected significantly more frequently in patients with synchronous multiple lesions $[2,3,8]$, a predictive indicator for metachronous recurrence would correspond with the indicator for synchronous multiple gastric carcinoma.

Wittekind et al. [14] analyzed 61 patients with synchronous gastric carcinoma from among 1664 patients, and suggested that multiple primary tumors arose from precancerous conditions leading to similar genetic alterations. It is generally accepted that IM in the stomach increases the risk of gastric cancer [26-29]. However, it has been suggested that only $0.1 \%-0.2 \%$ of IM is related to the carcinogenesis of intestinal-type gastric cancer worldwide [30]. Therefore, the IM significantly correlated with carcinogenesis of intestinal-type cancer should be selected for use as an appropriate marker. 


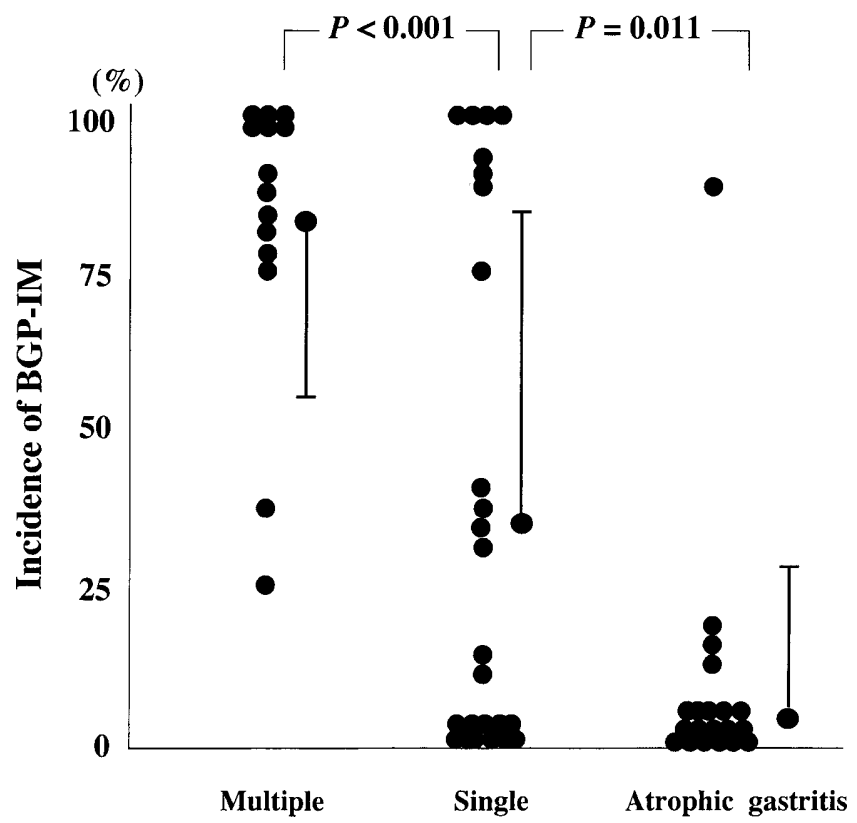

Fig. 4. Distribution of incidence of intestinal metaplasia expressing brain type glycogen phosphorylase $(B G P-I M)$ in multiple carcinomas, single-carcinoma, and atrophic gastritis groups. Multiple, Stomachs with multiple gastric carcinomas; single, stomachs with single gastric carcinomas; atrophic gastritis, stomachs with endoscopic atrophic gastritis

Our previous consecutive studies [17-21] revealed that the proportion of BGP positivity in both cancer and IM was significantly greater in the intestinal-type carcinoma than in the diffuse type $(P<0.001)$; also, we found that BGP-IM had a much stronger correlation with gastric carcinoma than the conventional type of incomplete-type IM, and BGP-IMs were significantly higher in a proliferating state than in those samples without BGP $(P<0.05)$, and p53 mutation occurred only in BGP-IM, suggesting that BGP-IM is a precancerous condition for intestinal-type carcinoma. Thus, our findings indicate that BGP-IM is an excellent marker of the early stage of gastric carcinogenesis.

The present study clearly showed that the incidence of BGP-IM appearance was significantly more frequent in the stomachs with multiple gastric carcinomas than in those with single carcinoma or those with atrophic gastritis $(P<0.001)$. The finding that some of the stomachs with a single carcinoma had a high incidence of BGP-IM may suggest the coexistence of microscopic intestinal-type carcinomas or the possibility of metachronous recurrence in the future. Assay of BGP in IM by immmunohistochemistry in endoscopic biopsy specimens is an easy and reliable technique to assess BGP-IM status in the stomach, and thus could serve as a predictor of the high potential of a stomach in which synchronous gastric carcinoma coexists for generating metachronous gastric carcinoma. These results suggest that the analysis of BGP expression in IM in biopsy specimens will contribute to the pre- and postoperative assessment of multiple and metachronous gastric cancer.

Most gastric cancers of the intestinal type are known to occur on the distal side of the endoscopic atrophic border $[15,16]$. We agree with both the opinion that "the surgeon is required to resect the area including the F-line at the time of distal gastrectomy so as not to leave another cancer in the gastric remnant" [15] and the opinion that "the treatment of multiple gastric cancer does not require extended operative procedure, and EMR may be indicated if each lesion fits the criteria for treatment and careful follow-up is ensured" [31]. The important thing is to have a good predictor for metachronous recurrence after local treatment $[8,25]$. The present study demonstrated that BGP-IM was detected even in the stomachs with endoscopic atrophic gastritis without any malignant lesion, suggesting that BGP-IM was not a pathological entity which was associated with the change of a carcinogenic microenvironment in the gastric mucosa. Therefore, BGP can serve as a potential predictor for the risk-assessment of the development of multiple and/or metachronous carcinomas. It may be possible to follow-up new lesions by this method, and follow-up studies will give better information on whether BGP-IM positivity could be a good predictor for metachronous recurrence after local treatment.

In conclusion, we demonstrated the importance of BGP-IM as a predictor for the metachronous recurrence of gastric carcinoma, and we propose immunohistochemical staining of BGP in multiple endoscopic biopsy specimens as a predictive indicator of synchronous cancer and/or metachronous recurrence.

Acknowledgment This work was supported by Grantsin-Aid (nos.11671254 and 12877194) for Scientific Research from the Ministry of Education, Japan.

\section{References}

1. Tada M, Murakami A, Karita M, Yanai H, Okita K. Endoscopic resection of early gastric cancer. Endoscopy 1993;25:445-50.

2. Takekoshi T, Baba Y, Ota H, Kato Y, Yanagisawa A, Takagi K, et al. Endoscopic resection of early gastric carcinoma: results of a retrospective analysis of 308 cases. Endoscopy 1994;26:352-8.

3. Kojima T, Parra-Blanco A, Takahashi H, Fujita R. Outcome of endoscopic mucosal resection for early gastric cancer: review of the Japanese literature. Gastrointest Endosc 1998;48:550-4.

4. Ohgami M, Kumai K, Otani Y, Wakabayashi G, Kubota T, Kitajima M. Laparoscopic wedge resection of the stomach for early gastric cancer using a lesion-lifting method. Dig Surg 1994; 11:64-7.

5. Roukos DH. Current advances and changes in treatment strategy may improve survival and quality of life in patients with potentially curable gastric cancer. Ann Surg Oncol 1999;6:46-56. 
6. Janssen CW Jr, Lie RT, Maartmann-Moe H, Matre R. Who gets a second primary cancer after gastric cancer surgery? Eur J Surg Oncol 1990;16:195-9.

7. Seto Y, Nagawa H, Muto T. Treatment of multiple early gastric cancer. Jpn J Clin Oncol 1996;26:134-8.

8. Arima N, Adachi K, Katsube T, Amano K, Ishihara S, Watanabe $\mathrm{M}$, et al. Predictive factors for metachronous recurrence of early gastric cancer after endoscopic treatment. J Clin Gastroenterol 1999;29:44-7.

9. El-Zimaity HM, Ota H. Endoscopic resection for early gastric cancer: possibilities and limitations. J Clin Gastroenterol 1999; 29:5-6.

10. Honmyo U, Misumi A, Murakami A, Haga Y, Akagi M. Clinicopathological analysis of synchronous multiple gastric carcinoma. Eur J Surg Oncol 1989;15:316-21.

11. Kosaka T, Miwa K, Yonemura Y, Urade M, Ishida T, Takegawa $\mathrm{S}$, et al. A clinicopathological study on multiple gastric cancers with special reference to distal gastrectomy. Cancer 1990;65:26025 .

12. Brandt D, Muramatsu Y, Ushio K, Mizuguchi Y, Itabashi M, Yoshida S, et al. Synchronous early gastric cancer. Radiology 1989;173:649-52.

13. Kodera Y, Yamamura Y, Torii A, Uesaka K, Hirai T, Yasui K, et al. Incidence, diagnosis and significance of multiple gastric cancer. Br J Surg 1995;82:1540-3.

14. Wittekind C, Klimpfinger M, Hermanek P, Tannapfel A. Multiple simultaneous gastric carcinomas. Br J Cancer 1997;76:1604-9.

15. Kimura K, Takemoto T. An endoscopic recognition of the atrophic border and its significance in chronic gastritis. Endoscopy 1969;1:87-97.

16. Yoshimura T, Shimoyama T, Fukuda S, Tanaka M, Axon AT, Munakata A. Most gastric cancer occurs on the distal side of the endoscopic atrophic border. Scand J Gastroenterol 1999;34:107781.

17. Shimada S, Maeno M, Misumi A, Akagi M. Histochemical study of phosphorylase in proliferating cells of intestinal metaplasia and carcinoma of the human stomach. Scand J Gastroenterol 1984; 19:965-70.

18. Shimada S, Honmyo U, Yagi Y, Ikeda T, Ogawa M, Yokota T. Expression of glycogen phosphorylase activity in minute gastric carcinoma. Am J Gastroenterol 1992;87:1230-1.

19. Shimada S, Maeno M, Misumi A, Takano S, Akagi M. Antigen reversion of glycogen phosphorylase isoenzyme in carcinoma and generative zone of intestinal metaplasia of the human stomach. Gastroenterology 1987;93:35-40.

20. Matsuzaki H, Shimada S, Uno K, Tsuruta J, Ogawa M. Novel subtyping of intestinal metaplasia in the human stomach: braintype glycogen phosphorylase expression in the generative zone and its relationship with carcinogenesis. Am J Clin Pathol 1998;109:181-9.

21. Shimada S, Tashima S, Yamaguchi K, Matsuzaki H, Ogawa M. Carcinogenesis of intestinal-type gastric cancer and colorectal cancer is commonly accompanied by expression of brain (fetal)type glycogen phosphorylase. J Exp Clin Cancer Res 1999;18:1118.

22. Suzuki S, Suzuki H, Endo M, Takemoto T, Kondo T. Endoscopic diagnosis of early cancer and intestinal metaplasia of the stomach by dyeing. Int Surg 1973;58:639-42.

23. Ida K, Hashimoto Y, Kawai K. In-vivo staining of gastric mucosa. Endoscopy 1975;7:18-24.

24. Ignacio PC, Baldwin BA, Vijayan VK, Tait RC, Gorin FA. Brain isozyme of glycogen phosphorylase: immunohistochemical localization within the central nervous system. Brain Res 1990;529:429.

25. Kawamura A, Adachi K, Ishihara S, Katsube T, Takashima $\mathrm{T}$, Yuki M, et al. Correlation between microsatellite instability and metachronous disease recurrence after endoscopic mucosal resection in patients with early stage gastric carcinoma. Cancer 2001; 91:339-45.

26. Morson BC. Intestinal metaplasia of the gastric mucosa. $\mathrm{Br} \mathbf{J}$ Cancer 1995;85:377-85.

27. Ming SC, Goldman H, Freiman DG. Intestinal metaplasia and histogenesis of carcinoma in human stomach. Cancer 1967;20: 1418-29.

28. Stemmermann GN. Intestinal metaplasia of the stomach. Cancer 1994;74:556-64.

29. Correa P, Shiao Y. Phenotypic and genotypic events in gastric carcinogenesis. Cancer Res 1994;54:1941-3.

30. Kubo T. Etiology of intestinal metaplasia. In: Takemoto T, Kawai K, Ida K, Suzuku S (eds). Intestinal metaplasia of the human stomach (in Japanese). Tokyo: Igaku-Tosho-Shuppan; 1981. p. 27-31.

31. Takeshita K, Tani M, Honda T, Saeki I, Kando F, Saito N, et al. Treatment of primary multiple early gastric cancer: from the viewpoint of clinicopathologic features. World J Surg 1997;21: 832-6. 